\title{
Gestão de material médico-hospitalar e o processo de trabalho em um hospital público
}

\author{
Medical-hospital material management and the working process in a public hospital \\ Gestión de material médico-hospitalario y el proceso de trabajo en un hospital público
}

\section{Simone Domingues Garcia', Maria do Carmo Lourenço Haddad", Mara Solange Gomes Dellaroza", Daniele Bernardi da Costa"', Juranda Maia de Miranda ${ }^{\text {IV }}$}

\author{
' Universidade Estadual de Londrina, Residência em Gerência dos Serviços de Enfermagem. Londrina-PR, Brasil. \\ "Universidade Estadual de Londrina, Departamento de Enfermagem. Londrina-PR, Brasil. \\ "' Universidade de São Paulo, Escola de Enfermagem de Ribeirão Preto. (Mestranda) Ribeirão Preto-SP, Brasil. \\ Iv Hospital Dr. Eulalino Ignácio de Andrade, Gerência de Enfermagem. Londrina-PR, Brasil.
}

Submissão: 26-03-2011 Aprovação: 08-04-2012

\section{RESUMO}

Estudo exploratório-descritivo de abordagem qualitativa. Objetivou-se identificar a percepção sobre a gestão de material médico-hospitalar no processo de trabalho de profissionais em hospital público de média complexidade. As informações foram levantadas utilizando a questão norteadora: como a gestão de materiais influencia o seu processo de trabalho? A análise dos relatos permitiu identificar cinco categorias denominadas: ausência de autonomia na escolha dos materiais, falta de manutenção de equipamentos, burocracia no processo de compra, falta de qualidade e ausência de envolvimento dos profissionais na gestão de materiais. Concluiu-se que a gestão de materiais é centrada na direção de enfermagem, necessitando de maior participação dos outros profissionais na escolha dos materiais em uso, para reduzir as fragilidades encontradas nesse processo.

Descritores: Enfermagem; Gerência; Materiais.

\section{ABSTRACT}

This article presents an exploratory-descriptive study structured in a qualitative approach. The main objective was to identify the perception of medical-hospital materials on the work process of professionals in a medium complexity public hospital. Information has been collected using the research question: How does the materials management process influence work process? The report analysis has identified five different categories: lack of autonomy in the choice of materials, lack of maintenance of equipment, bureaucracy in the purchasing process, poor quality and absence of professional involvement in the materials management. It is concluded that the materials management is focused on the nursing direction, which requires greater involvement of other professionals in the materials choice used to reduce the weakness in that process.

Key words: Nursing; Management; Material.

\section{RESUMEN}

Este es un estudio exploratorio descriptivo con abordaje cualitativo. Objetivó-se identificar la percepción de la gestión de materiales médico hospitalarios en el proceso de los profesionales que trabajan en un hospital público de mediana complejidad. Se ha obtenido la información mediante la pregunta: ¿Cómo influye la gestión de materiales en su proceso de trabajo? El informe del análisis ha permitido identificar cinco categorías denominadas: la falta de autonomía en la elección de los materiales, la falta de mantenimiento de los equipos, la burocracia en el proceso de compra, la mala calidad y la falta de envolvimiento de los profesionales en la gestión Se ha encontrado que la gestión de materiales está centrada en la dirección de la enfermería, lo que requiere una mayor participación de otros profesionales en la elección de los materia les en uso, buscando así la reducción de las debilidades en ese proceso.

Palabras clave: Enfermería; Gestión; Materiales. 


\section{INTRODUÇÃO}

No processo gerencial na área da saúde um dos setores mais complexos e de maior custo, que exige constantes atualizações devido as mudanças e surgimentos de novos produtos é a área de gestão de materiais hospitalares ${ }^{(1)}$. Materiais são considerados insumos ou fatores produtivos, de natureza física, com determinada durabilidade, empregados na realização de procedimentos/atividades assistenciais aos pacientes. A escassez de alguns destes, considerados imprescindíveis para assistência é um dos pontos que mais afligem os gestores dos serviços de enfermagem ${ }^{(2)}$.

A gestão de materiais é um processo no qual se planeja, executa e controla, em condições mais eficientes e econômicas, o fluxo de materiais, partindo das especificações dos artigos a comprar até a entrega do produto ${ }^{(3)}$. Os avanços tecnológicos têm impulsionado o aumento constante da complexidade assistencial, exigindo um nível de atenção cada vez mais elevado, por parte dos profissionais de saúde, criando uma demanda crescente por recursos materiais ${ }^{(4)}$

A administração de recursos materiais tem sido motivo de preocupação nas organizações de saúde, tanto nas do setor público, como no privado, que fazem parte da rede complementar do Sistema Único de Saúde (SUS). As do setor público, devido a orçamentos restritos, necessitam de maior controle do consumo e dos custos para que não privem funcionários e pacientes do material necessário ${ }^{(4)}$.

A atuação do enfermeiro na administração de recursos materiais constitui-se uma conquista nas esferas de tomada de decisão, destacando a importância do seu papel na dimensão técnico-administrativa inerente ao processo de cuidar e gerenciar $^{(4)}$. É válido ressaltar que a aquisição de materiais em instituições públicas segue a lei 86.666/1993, que norteia as licitações ${ }^{(6)}$.

Ao considerar as afirmações descritas e pela análise do cenário atual envolvendo a gestão de material médico-hospitalar, justifica-se como relevante e essencial para o desenvolvimento técnico- científico do enfermeiro maiores estudos sobre o assunto. O despertar inicial do pesquisador na área surgiu a partir da observação da prática diária, que considera fundamental o conhecimento dos materiais utilizados para assistir os pacientes com segurança e qualidade. $\mathrm{O}$ número de publicações com o tema ainda é escasso no Brasil dado a sua importância em todos os âmbitos da saúde, podendo ser visto como estratégia de estímulo na produção de artigos por enfermeiros.

Com isso, o estudo utilizou a seguinte questão norteadora para embasar a pesquisa: Qual a influência da gestão de material médico-hospitalar no seu processo de trabalho?

\section{MÉTODO}

Trata-se de um estudo descritivo, exploratório de natureza qualitativa. As abordagens qualitativas conformam-se melhor a investigações de grupos e segmentos delimitados e focalizados, de histórias sociais sob a ótica dos atores, de relações e para análises de discursos e de documentos Utilizou-se para a coleta dos dados a técnica de entrevista que oferece informações referentes a fatos, idéias, crenças, maneira de pensar; opiniões, sentimentos, entre outros ${ }^{(7)} \mathrm{O}$ roteiro para esse tipo de entrevista tem como finalidade direcionar a interlocução e deve ser construído de modo que permita flexibilidade nas conversas e a absorção de novos temas e questões interpostas pelo interlocutor, constitutivas de sua estrutura de relevância(7)

O estudo foi realizado em um hospital público de média complexidade, integrado ao Sistema Único de Saúde e localizado na região norte do Paraná. Presta serviço de Pronto-Atendimento nas clínicas médica, cirúrgica e pediátrica. $\mathrm{O}$ serviço de internação conta com 14 leitos pediátricos, 20 leitos da clínica médica, e dez leitos de clínica cirúrgica. O serviço de pronto-atendimento possui 15 leitos de observação, dez de internação e duas salas de emergências correspondendo a quatro leitos. Possui serviço de cirurgias eletivas de médio e pequeno porte.

O estudo foi desenvolvido em duas etapas, sendo que na primeira realizou-se a análise de documentos referentes a gestão dos recursos materiais disponíveis em manuais, ofícios, regulamentos, leis, entre outros. A segunda etapa constitui-se na realização de entrevistas semi-estruturadas com trabalhadores envolvidos no processo de gestão e utilização de material médico hospitalar.Realizou-se em julho de 2010 com 19 profissionais atuantes, envolvendo enfermeiros, técnicos de enfermagem, técnicos administrativos e auxiliares operacionais.

Os critérios de inclusão no estudo era que o trabalhador participasse diretamente da padronização, especificação e previsão de material médico-hospitalar, e que após o convite para sua participação concordasse em assinar o Termo de Consentimento Livre e Esclarecido, contendo todas as informações importantes da pesquisa para o conhecimento dos atores envolvidos. Participaram do estudo dois enfermeiros gerenciais, sendo um responsável pela gestão do serviço de enfermagem e o outro pelo processo de aquisição de materiais, sete enfermeiros supervisores dos setores de internação, pronto atendimento e centro cirúrgico; dois técnicos administrativos responsáveis pela compra e pela recepção dos materiais no almoxarifado; dois auxiliares operacionais que solicitavam os materiais para uso nos setores; seis técnicos de enfermagem que armazenavam e utilizavam os materiais sendo, dois de cada turno de trabalho, totalizando 19 profissionais envolvidos no processo de gestão de material médico-hospitalar.

A percepção dos entrevistados foi obtida por meio da seguinte questão norteadora: Qual a influência da gestão de material médico-hospitalar no seu processo de trabalho? Após a coleta os dados foram transcritos e analisados buscando encontrar através da análise dos discursos seu real significado para os atores envolvidos ${ }^{(7)}$ Para garantir o anonimato dos trabalhadores, substituímos os nomes por iniciais da categoria profissional e assim sucessivamente, sendo EG (enfermeiro gerente); EA (enfermeiro assistencial) em uma numeração de um a sete; EM (enfermeiro que realiza compra dos materiais); TE (técnico de enfermagem) seguindo numeração de um a seis; TA (Técnico administrativo) um e dois e AO (auxiliar operacional) um e dois, formando assim unidades de significado de acordo com o cargo desempenhado. 
O projeto de pesquisa foi aprovado pelo comitê de ética em pesquisa com cadastro no Sistema Nacional de Informações sobre Ética em Pesquisa com CAAE nº 0094.0.268.000-10.

\section{RESULTADOS E DISCUSSÃO}

Apresentamos os resultados em duas etapas, sendo a primeira em forma de fluxograma (Figura 1) onde é demonstrado o processo de gestão de material médico-hospitalar na instituição onde o estudo foi realizado; e na segunda etapa apresentamos a análise e discussão dos dados coletados nas entrevistas que foram classificadas em cinco categorias.

Após análise das entrevistas formou-se cinco categorias que expressam a influência da gestão de material médico-hospitalar no processo de trabalho dos atores envolvidos, sendo: 1) Ausência de autonomia na escolha do material médico-hospitalar; 2) Falta de manutenção de equipamentos e material médico-hospitalar; 3) Burocracia no processo

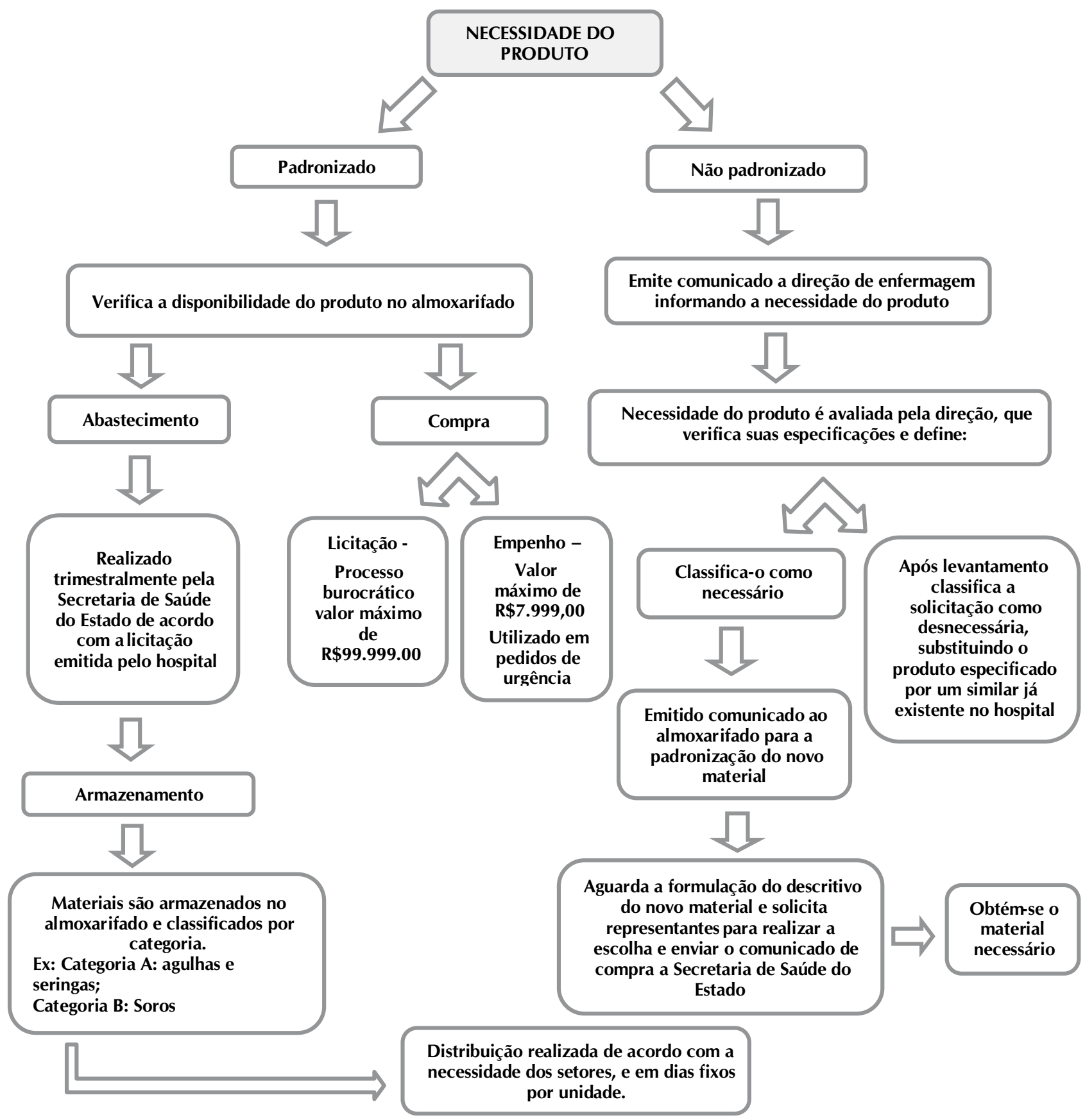

Figura 1 - Fluxograma do processo de gestão de material médico-hospitalar em hospital público de média complexidade.

Londrina-PR, 2010. 
de compra; 4) Falta de qualidade de alguns materiais; e 5) Ausência de envolvimento profissional na gestão de material médico-hospitalar.

As categorias formadas envolvem aspectos positivos e negativos do processo de trabalho, respeitando o significado expressado pelos profissionais durante o vivenciar de sua tarefa.

\section{Ausência de autonomia na escolha do material médico -hospitalar}

Nessa categoria as falas dos atores são claras em demonstrar que há centralização na tomada de decisão do profissional que compra os materiais, caracterizando o sentimento de submissão quanto ao poder de decidir qual seria a melhor escolha, conforme expresso nos relatos abaixo:

Dificuldade é que você não tem a oportunidade de escoIher o material tem que aceitar o que ele manda. Falta autonomia. (E.A)

Enfermeiro do setor deveria participar do processo licitatório porque nós que sabemos se o material é bom ou não. (E.A)

Torna-se visível ao analisar essa categoria a dicotomia existente nas funções gerenciais e assistenciais do enfermeiro. É necessário que o enfermeiro hospitalar tenha uma visão holística da assistência prestada e desenvolva competências gerenciais, demonstrando que a dicotomia existente entre assistência e gerência é algo desfavorável ao desenvolvimento do processo de trabalho(8)

A formação acadêmica e científica oferecida durante a graduação de enfermagem, é ineficiente em capacitar o futuro profissional no gerenciamento de material médico-hospitalar. Isso demonstra que o profissional deve procurar conhecimentos do assunto além da sua formação, considerando que a qualidade e quantidade do material é um dos aspectos que garante a eficiência do cuidado prestado(8)

A realidade é que não é possível oferecer uma assistência de qualidade se não houver uma integração entre as ações gerenciais e as assistenciais, e que o enfermeiro necessita atualizar-se quanto aos aspectos essenciais em uma unidade, como a escolha do material médico-hospitalar.

Outro fator relevante observado durante a entrevista com o enfermeiro responsável pelo processo de compras, foi que este profissional desconhece as especificações de alguns materiais, interferindo com isso na escolha de produtos específicos para determinados setores, principalmente os mais especializados como pediatria e centro cirúrgico.

Pessoas envolvidas não entendem materiais diferenciados no centro cirúrgico. (E.A)

Comprar para cozinha, para o laboratório é responsabilidade do auxiliar administrativo e o enfermeiro que realiza o pedido de compra desconhece o produto, não tem noção, quem pede não entende, compra errôneo, não sabe exatamente o que quer. (E.M)
É importante ressaltar que o processo de gestão de suprimento de material médico-hospitalar não tem recebido o devido trato profissional, desvalorizando-se o setor, por conseguinte a empresa hospitalar. Por vezes, delega-se essa responsabilidade a colaboradores não qualificados para o exercício da função. Os conceitos básicos, a linguagem técnica e os conhecimentos necessários devem ser adquiridos através de cursos especializados, o que resulta em ganhos de qualidade, produtividade, investimento no capital humano, eficiência e eficácia ${ }^{(9)}$

Ao enfermeiro, como líder de equipe, compete à decisão de ações que envolvem diversos profissionais, decisões essas tomadas muitas vezes individualmente, influenciando o resultado final. A responsabilidade frente à instituição e suas normas é oferecer o melhor serviço possível, sendo de sua competência se envolver em conhecimentos de outras áreas se esses estão dentro de suas tomadas de decisões.

\section{Falta de manutenção de equipamentos e material médico- -hospitalar}

Durante as entrevistas o conceito de materiais e equipamentos não apresentou diferenciação, demonstrando que os profissionais consideram que materiais envolvem desde os de consumo até os equipamentos permanentes. Relataram que um dos principais problemas enfrentados é o mau uso dos equipamentos e a falta de manutenção, influenciando diretamente na qualidade da assistência oferecida.

Expressaram que é lento o processo de manutenção corretiva e ausente o preventivo, sendo realizados consertos dos materiais permanentes somente quando não há condições de uso, passando então a necessitar de tempo maior para seu conserto, influenciando diretamente na qualidade da assistência prestada.

A manutenção é demorada e não há manutenção preventiva regular. (T.E) (E.A)

Demora muito os consertos dos equipamentos. (T.E)

A escassez de equipamentos necessários e específicos também foi referida como aspecto negativo para a execução do trabalho.

[...] falta tudo[ ] se tivesse número adequado seria mais rápido mais fácil. (E.A)

A presença de grandes estoques de alguns materiais e a escassez de outros, dentro de um hospital, é talvez um dos pontos que mais afligem os profissionais envolvidos com o processo gerencial. A escassez implica muitas vezes na interrupção da assistência, levando a vivência de situações danosas e estressantes para o cliente, família e profissionais ${ }^{(10)}$.

Os almoxarifados hospitalares, cuja função é o gerenciamento de estoques, não desenvolvem suas atividades com eficiência e eficácia, não controlando corretamente o fluxo de materiais. Os motivos mais apontados, entre outros, que levam a essa dificuldade de controle, são: a diversidade de materiais e a falta de informatização nesse setor ${ }^{(10)}$. 
Como ponto positivo e diferenciado da instituição em estudo é que a mesma possui sistema informatizado para a gestão de materiais, otimizando o tempo do funcionário que mantém as informações atualizadas em tempo real, como a quantidade de materiais no estoque sem ter que realizar essa tarefa manualmente.

O programa de gestão de materiais informatizado facilitou o trabalho, não precisa mais escrever os manuais, se precisar de relatórios tem na hora [...] (T. A)

Para otimizar o uso das informações nesse setor sugere-se uma adequação no sistema de informatização para que se utilize todas as funções do programa relacionadas a gestão de materiais, como a conecção entre os setores para que o pedido de material possa ser elaborado via sistema, conseguindo com isso agilizar o processo de trabalho, obtendo maior confiabilidade nas informações.

A falta de conscientização do uso dos materias também foi verificada, pois não há na instituição um treinamento ou um processo de sensibilização da importância do uso correto dos materiais e equipamentos.

Repetidas vezes o serviço de manutenção tem que realizar nova compra do equipamento. (E.M)

O cuidado com os materiais permanentes na instituição em estudo e em todas as organizações de saúde é essencial para que o patrimônio seja preservado e obtenha durabilidade, já que foram projetados para serem usados por um tempo prolongado. Devido ao mau uso desses materiais, foi notável o aumento nas solicitações de compras/consertos de determinados equipamentos, fazendo com que equipamentos semi-novos ficassem parados e inutilizados a espera de um novo processo de compra.

O processo de cuidar nas unidades especializadas depende em parte do uso racional e seguro de equipamentos de suporte à vida, entre outros procedimentos. Sabe-se, entretanto que, para garantir a qualidade do cuidar com vistas à longevidade dos equipamentos, faz-se necessário uma política de manutenção efetiva ${ }^{(11)}$.

Torna-se imprescindível também o conhecimento das normas técnico-operacionais dos equipamentos pelos profissionais de saúde uma vez que os equipamentos são importantes ferramentas no seu processo de trabalho ${ }^{(11)}$.

Há confirmação em estudo(12) que as enfermeiras constituem-se em um nível decisório importante na alocação de recursos, quando decidem em suas unidades de trabalho as prioridades de seus serviços, decidem quem e quanto tempo será despendido nos cuidados, e quais recursos serão empregados. Essas decisões afetam diretamente no número e na qualidade dos atendimentos.

O processo de educação continuada proporciona uma redução significativa de custos com manutenção e de satisfação no trabalho por possuir mais materiais disponíveis para uso, visto que menor quantidade de materiais necessitarão de manutenção de urgência(13).

\section{Burocracia no processo de compra}

O processo de compra no campo de pesquisa é feito por meio de licitações, que são procedimentos administrativos mediante o qual a organização pública seleciona a proposta mais vantajosa para o contrato de seu interesse, visando proporcionar oportunidades iguais aos fornecedores. As modalidades de licitação utilizadas para a compra de materiais nas instituições são a concorrência, a tomada de preço e o convite. A escolha de uma ou outra modalidade de licitação ocorre em função do valor estimado para a compra e dos casos de emergência, em que a falta de material pode ocasionar prejuízos no atendimento ao paciente ${ }^{(14)}$.

É necessário as instituições públicas respeitarem rigidamente as normas legais que regulamentam as licitações e os critérios e detalhes do processo, considerando a importância de existir um controle ao adquirir materiais para uma organização, porém em muito casos torna-se moroso e requer um tempo maior de espera pelo produto.

A lentidão nos processos administrativos presenciada nos serviços de saúde no Brasil é apontada como problema crônico, gerando formalidade nas comunicações, excessiva burocracia, apresentando serviços que não satisfazem os colaboradores externos ${ }^{(9)}$

Tudo passa por licitação, não pode escolher o material, ganha o menor preço; tempo de espera pelo material; não tem acesso ao material antes de chegar, já chega comprado. (E.A)

A aquisição de um produto de qualidade é um processo demorado. (T.E)

Informalmente, o enfermeiro sempre participou do processo de compras de materiais em hospitais, tendo em vista que sua competência lhe assegura condições para opinar quanto ao tipo, a quantidade, a qualidade dos materiais a serem adquiridos para utilização nas enfermarias. Atualmente, o enfermeiro tem atuado em comissões de licitação, integrando os grupos de assessoria de compra e emitindo pareceres técnicos nos processos de compra; e alguns serviços de enfermagem em instituições de saúde de grande porte, tem criado em sua estrutura, assessorias, seções ou setores de enfermagem para a execução de funções como controle de qualidade, seleção e compra de materiais utilizados na assistência ao cliente ${ }^{(14)}$.

O aumento da participação do enfermeiro tem se tornado notável, melhorando a qualidade e a quantidade dos produtos porém não alterando a morosidade da ação de compras, visto ao fator de licitação ser algo que não remete a governabilidade do enfermeiro.

Nas instituições privadas, a compra é direta, isto é, o setor de compras negocia livremente com os fornecedores, seguindo apenas as diretrizes financeiras da instituição. É um sistema desburocratizado, e por isso, torna-se mais ágil ${ }^{(14)}$.

Percebe-se que o processo de aquisição de material médico hospitalar adotado na instituição em estudo respeita as normas preconizadas pelo SUS, porém nota-se que algo instituído pelo poder público também possui fragilidades, como a morosidade na aquisição de materiais. 
Torna-se oportuno a discussão de uma atualização no processo licitatório público, em busca de melhorar o tempo hábil do processo de compra de materiais hospitalares e apresentar novas estratégias para o fortalecimento da qualidade de assistência a saúde.

Na instituição em estudo o cálculo da estimativa de materiais necessários é feito por meio de um programa de computador. O programa avalia o número utilizado nos últimos três meses e com uma margem de segurança calculada pelo próprio programa, que aproxima-se de $15 \%$ a mais de produtos, apresenta o número necessário para os próximos três meses. Os entrevistados relataram pontos positivos nesse processo, conforme observado nos relatos abaixo:

Fazemos planejamento com visão de futuro, para os próximos três meses. (E.M)

\section{Planejamos estoque trimestral. (E. G)}

O planejamento das ações em enfermagem e em saúde é fundamental para a sistematização do processo de trabalho das organizações e serviços e reflete na qualidade da assistência aos usuários. Não há garantia de sucesso, ou seja, os resultados não serão sempre positivos simplesmente por terem sido planejados, mostrando que planejamento é também um processo dinâmico, ativo e deliberativo, mas que sem ele as chances de fracasso são maiores. No Brasil, o planejamento das ações em saúde está atrelado aos princípios doutrinários e organizativos do SUS ${ }^{(15)}$.

Os entrevistados relataram também que mesmo não participando da escolha dos materiais, possuem como apoio a ficha de avaliação da qualidade do produto antes do mesmo ser aceito na unidade, o que auxilia na intenção de evitar um produto de qualidade inferior.

Quando o material não é aprovado pode ser feita ficha de avaliação e recusar o produto[...]. (E.G)

Porém quando ocorre a recusa do material por considerá-lo de qualidade inferior ao desejado, o mesmo já encontra-se comprado com um número calculado de uso para os próximos três meses. Isso significa que será necessário utilizar todo o material em estoque para depois ser iniciado um novo processo licitatório para aquisição de outro produto, comprometendo diretamente a qualidade da assistência prestada.

\section{Falta de qualidade de alguns materiais}

A qualidade, de acordo com a Organização Mundial da Saúde OMS ${ }^{16}$ "define-se como um conjunto de elementos que incluem: um alto grau de competência profissional, a eficiência na utilização dos recursos, um mínimo de riscos, um alto grau de satisfação dos pacientes e um efeito favorável na saúde". A qualidade dos produtos disponíveis na instituição está diretamente ligada ao processo de aquisição e uso destes materiais.

Em relação à qualidade dos materiais os entrevistados referiram:
A qualidade se torna um defeito e tendo material em abundância, as pessoas desperdiçam. (T.E) (E.A)

\section{[...] usamos muitos materiais de má qualidade. (E.A) (T.E)}

Os entrevistados consideraram ser possível a aquisição de materiais de qualidade superior, porém é necessário realizar todas as fases do processo de compra, como uma delas o detalhamento do material, conforme apresentado na Figura 1.

Refere-se que a qualidade de um produto ou de um serviço é medida pelo conjunto de características capazes de atender as necessidades implícitas do cliente, que está ligada ao modo de ser de cada um, ao senso de observação e a sua realidade. Por sua vez, as necessidades explícitas estao relacionadas a aspectos objetivos expressos formalmente em contratos, especificações de projetos, manuais de operações de equipamentos. Por conseguinte, revelam o compromisso que o fornecedor assume junto ao cliente ${ }^{(17)}$.

Dentre as reformulações necessárias em busca da qualidade do material oferecido está a melhora nas especificações dos materiais padronizados e não padronizados. Com a especificação adequada é possível obter materiais de qualidade superior e consequentemente, melhorar a qualidade da assistência oferecida.

O monitoramento da eficácia dos produtos adquiridos para que consiga avaliar frequentemente o que está sendo alcançado com o serviço também é de extrema importância, pois apenas visualizando e vivenciado no dia-a-dia a disponibilidade de materiais é que torna-se possível a formação de uma visao crítica do assunto.

Abragendo a questão de desperdício de materiais, além do relato acima, a fala abaixo reforça a necessidade de garantir a qualidade dos materiais medico hospitalares:

As vezes o produto é mais barato, mas acaba sendo necessário utilizar três unidades, tornando relativamente mais caro. (T.E)

O serviço público considera que seus maiores problemas estão centrados na falta de recursos financeiros, porém é perceptível que a escassez de alguns materiais médico hospitalar juntamente com a sua má utilização, falta de profissionais qualificados e pouca atenção no planejamento logístico de insumos interfere na qualidade do que se dispõe ${ }^{(9)}$.

O hospital, dentre seus objetivos, tem por missão conseguir uma maior confiabilidade em todos os serviços em que oferece à população. Para alcançar suas metas, deve-se evitar todo tipo de desperdício: de material hospitalar; de esforços desnecessários, com o uso indevido do tempo; com o próprio trabalho empregado em uma atividade (quando há o retrabalho), com processos ineficientes entre tantos outros $^{(18)}$.

Para que haja um uso correto dos recursos públicos, a administração de materiais dá suporte ao controle do processo das atividades realizadas num hospital, para também evitar desperdícios e controlar os custos. Quando se refere 
à percepção que os funcionários têm do desperdício, o que vem à mente basicamente é uso de recursos de materiais hospitalares em primeiro plano, já que o gasto com eles é maior que os demais recursos, e dele também se faz maior uso devido à própria atividade da instituição(18).

Da otimização dos recursos materiais, em particular, resultará o menor custo gerido pelas instituições, e na maior produtividade e qualidade de suas atividades, na prestação de serviços à comunidade e numa melhor relação custo/benefício ${ }^{(18)}$. Destaca-se que cabe ao gestor da unidade adequar equipamentos, recursos materiais e processos de trabalho, além de manter a equipe continuamente informada sobre os valores de tais materiais, como mais uma forma de conscientização sobre racionalização de custos $^{(19)}$.

Considerando esse aspecto, uma da sugestões válidas a instituição em estudo seria a implantação de um instrumento que avaliasse os materiais e equipamentos antes de sua compra, para que os profissionais que vão utilizá-los em sua prática profissional possam emitir a sua opinião sobre o produto.

Também deve ser recomendado ao setor de compras, a organização de um portfólio qualificado de fornecedores de produtos, além de programas internos de conscientização sobre o uso racional dos materiais ${ }^{(19)}$.

O instrumento de avaliação e o portfólio são elementos educativos e facilitadores da análise técnica dos materiais, dando suporte aos enfermeiros dos setores no momento de questionar a qualidade dos materiais adquiridos.

\section{Ausência de envolvimento profissional na gestão de mate- rial médico-hospitalar}

A ausência de envolvimento profissional voltada para a gestão de material médico- hospitalar também foi destacada durante as falas dos entrevistados.

A enfermagem deveria estar mais envolvida com a gestão de materiais. (A.O)

Quase sempre a gente esta sem algum equipamento, e assim infelizmente não é dada tamanha importância ao assunto( $E . A)$.

Nota-se com esses relatos a necessidade do enfermeiro envolver-se nas ações da gestão de materiais para acrescentar o seu conhecimento ao processo. A falta de envolvimento com a gestão de recursos materiais pode ter contribuído para que a equipe de enfermagem assuma uma postura passiva frente a esse aspecto. Por outro lado, a equipe alimenta a expectativa de que os materiais adquiridos atendam as reais necessidades da assistência, sem que se percebam co-responsáveis pelo processo de escolha sobre o que adquirir, formas de armazenar, controle quantitativo e monitorização da eficácia dos produtos após a aquisição ${ }^{(17)}$.

As áreas de apoio a assistência, como no caso a área de gestão de materiais, não estão totalmente sincronizadas com as demais, assim como não estão suficientemente instrumentalizadas para administrar, isoladamente, os recursos materiais, de forma a atender as necessidades das áreas finalísticas ${ }^{(17)}$.

É de suma importância que os enfermeiros participem do processo de seleção e compra de materiais, pois têm como avaliar se a quantidade e a qualidade do produto condizem com as necessidades da clientela e de sua equipe, que deve ser treinada constantemente, a fim de evitar desperdícios e, por extensão, o aumento do custo hospitalar por uso indevido desses materiais ${ }^{(19)}$.

Observou-se que a falta de envolvimento dos profissionais da instituição em estudo foi por falta de conhecimento da importância e falta de programas educacionais relacionados ao tema.

A capacitação do enfermeiro para desenvolver o gerenciamento de materiais trata-se de prepará-lo para as tarefas que Ihe são direcionadas legalmente, ou seja, as tarefas que irá desenvolver seja em cunho hospitalar ou em unidades básicas de saúde. Além de capacitar profissionais de saúde com competências técnicas especializadas é necessário o desenvolvimento de pessoas comprometidas com o processo de gestão, que exige qualidades como a criatividade, inovação, intuição, emoção, capacidade de se relacionar e, principalmente, a capacidade de manter-se atualizado ${ }^{(20)}$.

Destacamos o anseio por novas propostas de ensino que englobem perpectivas inovadoras, idéias que consigam durante a construção do aprendizado demonstrar a relevância de capacitar o enfermeiro em temas como a gestão de materiais.

\section{CONSIDERAÇÕES FINAIS}

Os resultados demontraram que o processo de gestão de materiais na instituição em estudo, da forma como vem sendo desenvolvido, influencia de forma significativa no trabalho dos profissionais que atuam diretamente na assistência. Porém o processo só se torna perceptível quando ocorre algum evento negativo, como quantidade e qualidade insuficiente de materiais e falta de manutenção preventiva de equipamentos, não obtendo a valorização necessária pelos profissionais envolvidos.

Ressalta-se que há conhecimento dos princípios do SUS que norteiam os processos de compras de material médico-hospitalar, e que a busca por mudança sugere a valorização das peculiaridades da instituição pesquisada e levantamento de estratégias inovadoras que acrescentem qualidade ao processo, sem deixar de respeitar o que é preconizado para as instituições públicas.

A proposta de reformulação envolveria a qualidade dos materiais oferecidos, que em muitas situações por estarem atrelados ao menor preço, diminuem a chance do serviço de apresentar opções com qualidade superior, sugerindo assim a melhora no descritivo dos produtos durante o processo de compras e aumento do número de fornecedores envolvidos, priorizando o quisito qualidade.

A valorização do tema e conscientização da equipe torna-se essencial para alcançar mudança notável no desenvolvimento do trabalho, alcançando com isso benefícios ao produto final, no caso o cliente atendido. 


\section{REFERÊNCIAS}

1. Honório MT, Albuquerque GL. A gestão de materiais em enfermagem. Ciênc Cuid Saúde 2005; 4(3):259-68.

2. Mesquita SRAM, Anselmi ML, Santos CB, Hayashida M. Programa interdisciplinar de interação domiciliar de Marília-SP: custos de recursos materiais consumidos. Rev Latinoam Enferm 2005;13(4):555-61.

3. Francischini PG, Gurgel FA. Administração de materiais e do patrimônio. São Paulo: Pioneira Thonson; 2002.

4. Castilho V, Gonçalves VLM. Gerenciamento de recursos materiais. In: Kurcgant P. Coordenadora. Gerenciamento em enfermagem. Rio de Janeiro: Guanabara Koogan; 2005. p.157-70.

5. Brasil. Lei n. ${ }^{\circ} 86.666$, de 21 de junho de 1993. Regulamenta o art. 37, inciso XXI da Constituição Federal, institui normas para licitações e contratos da administração pública e dá outras providências. Diário Oficial da União 21 jun 1993.

6. Minayo MCS. O desafio do conhecimento: pesquisa qualitativa em saúde. 8. ed. São Paulo: Hucitec; 2004.

7. Peres AM, Ciampone MHT. Gerência e competências gerais do enfermeiro. Texto \& Contexto Enferm 2006;15(3):492-9.

8. Massaro M, Chaves LDP. A produção científica sobre gerenciamento em enfermagem hospitalar: uma pesquisa bibliográfica. Cogitare Enferm 2009;14(1):150-8.

9. Lourenço $K G$, CastilhoV. Classificação $A B C$ dos materiais: uma ferramenta gerencial de custos de enfermagem. Rev Bras Enferm 2006;59(1);52-5.

10. Madureira CR, Veiga K, Sant'ana AFM. Gerenciamento de tecnologia em terapia intensiva. Rev Latinoam
Enferm 2000;8(6);68-75.

11. Francisco IMF, CastilhoV. A enfermagem e o gerenciamento de custos. Rev Esc Enferm USP 2002;36(3):240-4.

12. Silva GM, Seiffert OMCB. Educação continuada em enfermagem: uma proposta metodológica. Rev Bras Enferm 2009;62(3):362-6.

13. Costal CMA, Guimarães RM. Considerações sobre a administração de recursos materiais em um hospital universitário. Rev Enferm UERJ 2004;12(2):205-10.

14. Lanzoni GMM, Lino MM, Luzardo AR, Meireles BHS. Planejamento em enfermagem e saúde: uma revisão integrativa da literatura. Rev Enferm UERJ 2009;17(3):430-5.

15. Organização Mundial da Saúde (OMS). Avaliação de programas de saúde: normas fundamentais para sua aplicação no processo de gestação para o desenvolvimento nacional de Saúde. Genebra: OMS; 1993.

16. Honório MT, Albuquerque GL. A gestão de materiais em enfermagem. Ciênc Cuid Saúde 2005; 4(3):259-68.

17. Guiomar TC. Aranha RWV. Estudo de um dos indicadores do custo da qualidade: o desperdício. Rev Adm Saúde 2004; 6(23):43-55.

18. Lopes LA, Dyniewicz AM, Kalinowski LC. Gerenciamento de materiais e custos hospitalares em UTI neonatal. Cogitare Enferm 2010;15(2):278-85.

19. Aguiar AB, Costa RSB, Weirich CF, Bezerra ALQ. Gerência dos serviços de enfermagem um estudo bibliográfico. Rev Eletrônica Enferm [periódico na internet]. 2005 [acesso em 15 nov 2010]; 7(3):318-26. Disponível em: http:// www.fen.ufg.br/revista/revista7_3/original_09.htm. 